\title{
BSE causing public alarm
}

\section{London}

THE UK Department of Health is to back a study to follow changes in the incidence in Britain of Creutzfeld-Jakob disease, a rare but fatal neurodegenerative disease of man. The disease belongs to the family of spongiform encephalopathies which includes bovine spongiform encephalopathy or BSE, the so-called 'mad cow disease', an outbreak of which has excited great attention in the British press. There are fears, as yet unproven, that people eating meat from infected cows could go on to develop Creutzfeld-Jakob disease.

The first of several thousand cases of bovine spongiform encephalopathy was diagnosed in November 1986 after cows were fed meal containing tissue from sheep infected with scrapie, another spongiform encephalopathy. It is now generally accepted that the two diseases are caused by the same infective agent, but a link between them and CreutzfeldJakob disease is more controversial.

The official opinion of the Ministry of Agriculture Fisheries and Food (MAFF) is that the risk of transmission of bovine spongiform encephalopathy to humans is very small: "Scrapie. . . has existed in the national flock (of sheep) for more than 250 years without any evidence of human health problems", says Keith Meldrum, MAFF's chief veterinary officer

Timothy Holt, of the Middlesbrough General Hospital, who has published a paper in the British Medical Journal on the possibility of a link between bovine spongiform encephalopathy and Creutzfeld-Jakob disease, acknowledges that the link is unproven, but he believes that the similarities between the two diseases have not received enough attention.

So far, there has been little success in isolating the infective agents causing spongiform encephalopathies. No specific nucleic acids have been found associated with the diseases, which is surprising if conventional viruses are responsible.

Some 10,000 cattle have been destroyed so far. Farmers receive only partial compensation.
One school of thought, championed by Stanley Prusiner of the University of California at San Francisco, argues that a protein called $\operatorname{PrP}$ is the infective agent, but his views are not widely shared. Researchers working at the Institute for Animal Health in Edinburgh have evidence that there are several different strains of scrapie, which suggests that some form of genetic material must be present.

It is in this climate of ignorance at the molecular level that the new epidemiological study is to be launched. Bob Will, of the Western General Hospital in Edinburgh, will lead the research. His team was involved with an earlier survey in England and Wales which collected data for the period 1970-85. The new survey will cover the whole of the UK and investigate the distribution of Creutzfeld-Jakob disease by place and occupation.

The study will be difficult to interpret as the disease has a long incubation period (from 18 months to more than 20 years). The researchers will need to look back at the previous life history of patients to find causative factors. Will says that his team will be looking for changes over time in the incidence of the disease. If there is a link with bovine spongiform encephalopathy, then an increase in the number of cases of Creutzfeld--Jakob disease should become apparent some time after the beginning of the cattle out-break.

The Department of Health's decision to support the study coincides with two separate announcements of additional funding for research into bovine spongiform encephalopathy itself. MAFF is to spend an extra $£ 6.1$ million on research on epidemiology and diagnosis over the next three years, in line with the recommendations of the Tyrrell committee report, published on 9 January. The Agricultural and Food Research Council will spend $£ 6.3$ million over the next three years, mainly on the molecular biology of the disease.

Peter Aldhous

\section{Product licensing by phone}

\section{San Francisco}

Normally, a Product License Application (PLA) to the Food and Drug Administration (FDA) consists of 20 to 50 volumes, each 3 inches thick, of reports, charts, graphs and numbers detailing technical specifications and clinical results. But when biotechnology company Genentech last month filed a PLA seeking limited marketing approval for recombinant gamma interferon, it sent in instead a set of computer disks. The application is one of the first under a pilot study called CAPLAR (Computer Assisted PLA Review) being run by the FDA in conjunction with the Pharmaceutical Manufacturers Association.

The Genentech filing seeks permission to market interferon gamma-1b to patients with chronic granulomatous disease, an inherited disorder characterized by the failure of the body's white blood cells to kill invading bacteria. Although the filing is not yet complete, it is expected to take up about five standard diskettes, already configured for the FDA's software.

The submission marks a first for the CAPLAR programme and the FDA's Center for Biologics Evaluation and Research (CBER), which evaluates the vast majority of genetically engineered drugs. But a few new drug applications have been filed electronically under a similar programme called CANDAR.

Such submissions will not eliminate paper: reviewers still want paper copies to read. But FDA scientists will no longer have to wade through thickets of paperwork to find the chart or numbers they need to examine, and the data can be manipulated without numbers being manually transferred from tables to computers. "Reviewers can look at the data on disks and analyse the data on their own computers as they want to", said Kathryn Zoon, director of CBER's Division of Cytokine Biology.

In CAPLAR's first trial, working out various kinks and bugs might take up more time than the usual procedure, she admitted. But she thinks the system will eventually speed things up.

Zoon said her division has also established an electronic mail link with Genentech to help mitigate the effects of the time difference between Washington and San Francisco and make it easier to get quick and complete answers to any questions that arise. Electronic filing is not a requirement for other companies, but she hopes it will become more common. "I think in the long run it will definitely make an impact on the way we do business", she said, adding that in the long run the new system will become necessary or "there'll be so much paper in the buildings that nobody will be able to move".
Robert Buderi 